\title{
The Control System for Ink Keys of Press Based on DSP Controller
}

\author{
Wei Zhaogang \\ Shandong Industrial Vocational College \\ Zibo,China \\ weizhaog@163.com
}

\begin{abstract}
We have designed the control system for ink keys of press based on DSP controller TMS320F2812. The operator can remotely control the position of any ink key of ink fountains with a high precision. The upper computer can communicate with any DSP through SCI to transmit the control instructions.
\end{abstract}

Keywords- DSP; ink fountain of press; control system; PID control policy; digital filtering

\section{I . INTRODUCTION}

Every ink fountain of press includes $10-20$ partitions, and any partition need be independently controlled by adjusting ink key. The single ink key is adjusted by the corresponding mechanical handle fixed on the partition in traditional method. Although the method is easy, it is impossible to attain high precision. There is no repeatability, and the worker should run back and forth from press and ajusting erea to finish the ajusting procedure. We have designed the control system for ink keys of press based on DSP controller to overcome the shortcomings of traditional method. We have fixed a micromotor on every partition, and the worker can remotely adjust any ink key accurately depending on the upper computer placed on the platform.

\section{II . THE HARDWARE COMPONENTS OF SYSTEM}

The control system consists of power circuit, EEPROM, AD sampling circuit, I/O controlling circuit, SCI interface circuit and upper computer.

The main controller is TMS320F2812 which is a DSP controller produced by TI. With $150 \mathrm{MHz}$ maximum working frequency, the Harvard pipeline technology and hardware multiplier, the most instruction is completed in one clock cycle. This fast controller with stable in performance is appropriate for the fast multi channel sampling and real-time computing. The controller has two SCI interfaces and one CAN interface to meet the requirements of data exchanging whit others. The chip X5043 is used for watch dog timer and EEPROM to preserve the user parameters.

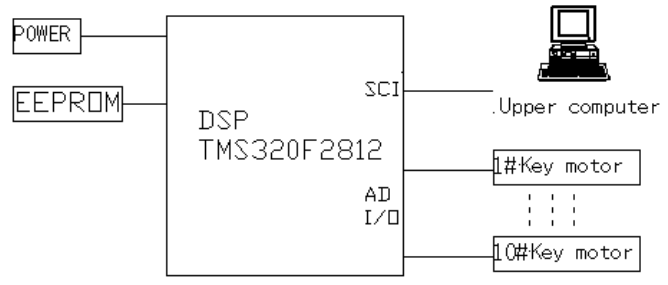

(fig.1 the structure of system)

\section{A .power circuit}

The external power supplies 5VDC power.
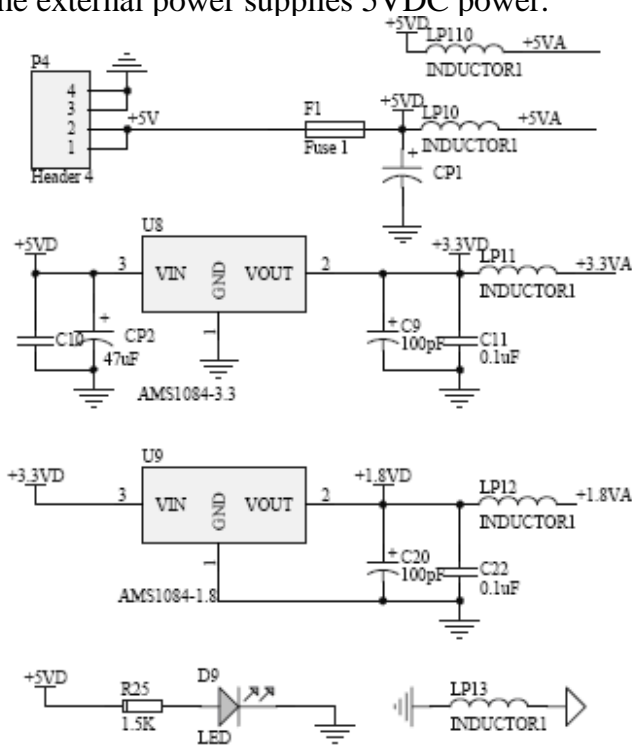

(fig.2 power circuit)

The working power of system includes 3.3VDC,1.8VDC and 3VDC used for sampling circuit. The main chips are AMS1084 series in power circuit as shown in fig.2.

\section{B .EEPROM circuit}

The chip X5043 is used to preserve user paremeters, which can communicate with CPU by SPI interface. And, basing on the chip including a watch dog timer, we can design a watch dog circuit easily. The circuit is shown in fig.3.

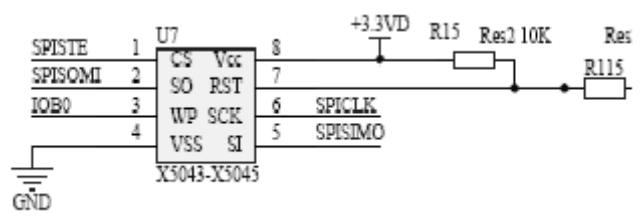

\section{(fig.3 EEPROM circuit)}

\section{AD sampling circuit}

Each motor board can control 24 motors. The CPU has a 16 channel ADC only, so a multiple switch is used. In order to improve the linearity of sampling circuit, the voltage follower is used in front of ADC. The circuit is shown in fig.4. 


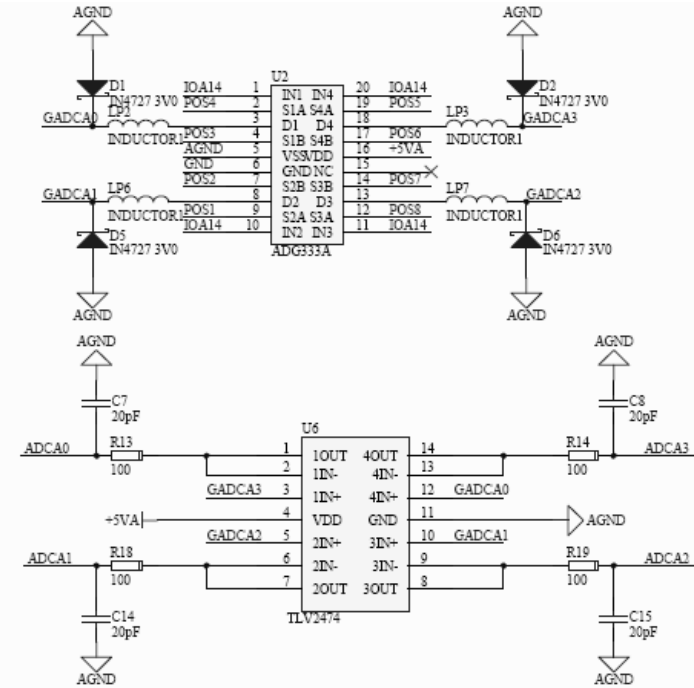

(fig.4 AD sampling circuit)

\section{SCI interface circuit}

The SCI interface is RS485. The main chip is MAX3485. The circuit is shown in fig.5.

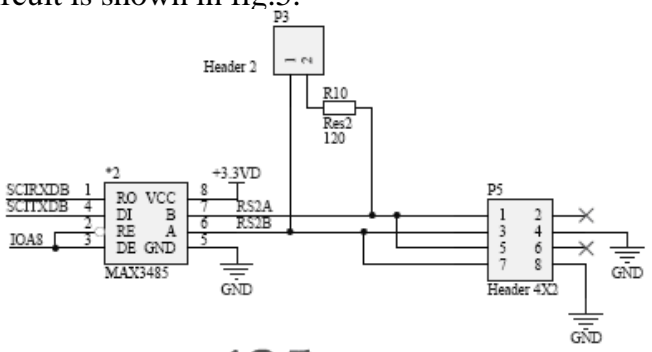

\section{5}

(fig.5 SCI interface circuit)

\section{THE SOFTWARES OF SYSTEM}

The DSP system can exchange datas with upper computer through RS485, the communication protocol is MODBUS. The DSP system obtains the position of each motor through $\mathrm{AD}$ circuit and sends the results to upper computer. Then upper computer sends working command to DSP system, and the DSP system control the motors running to reach the predefined position.

The programming language is $\mathrm{C}$, compiler environment is CCStudio. The software is designed according to the modular programming mode. The whole software consists of main program, AD sampling program, motor position controlling program and RS485 communication program.

\section{A.Main program}

The functions of main program includes: initializing all subsystems (initializing AD,initializing SCI,initializing SPI), waiting the

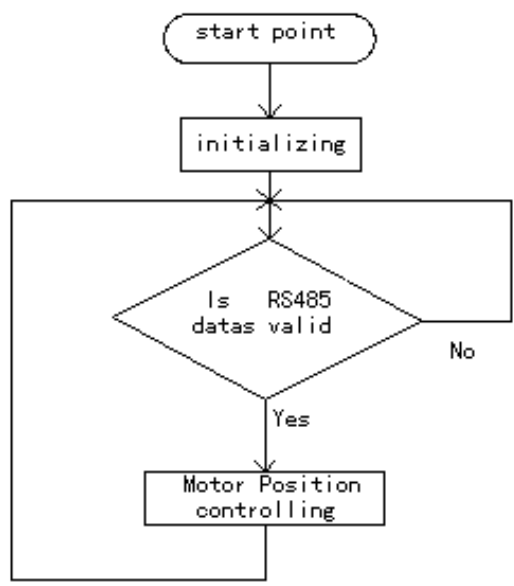

(fig.6 diagram of main program) respons of RS485, calling the motor position controlling program. The diagram of the program is shown in fig.6.

\section{$B$.AD sampling program}

The main chip TMS320F2812 has 16 high speed AD sampling channels, but the system should collect analog datas from 24 channels. So we have selected a multiple switch ADG33A. when sampling, the system collect 12 channels at a time, then other 12 channels. The alternant process is controlled by the multiple switch.

In order to obtain a high accuracy, we have used digital filter in the system. The filter used is the traditional arithmetic average value method. It is the simplest filter. But if the sampling frequency is appropriate, low-frequency harmonics can be filtered effectively.

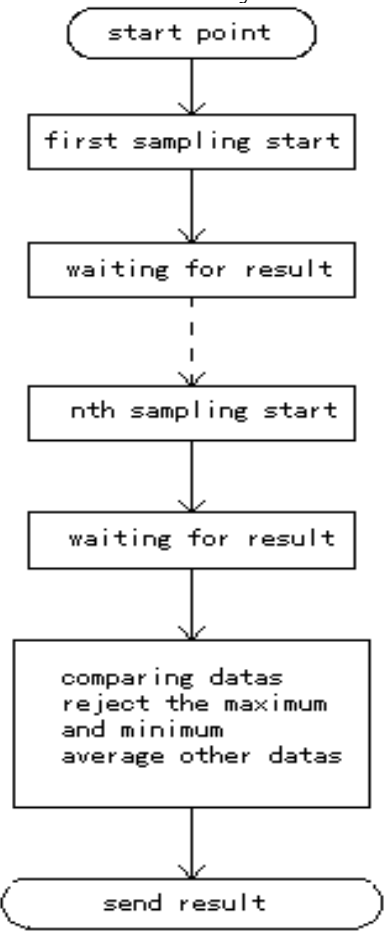

(fig.7 diagram of AD sampling program) 
Its shortcoming is that some obviously unreasonable data (such as mutant data caused by disturbance in a secular changed process ) is averaged as regular data. In order to overcome it, we reject the datas which have obvious deviation in

comparison with others in filter program with using higher sampling frequency. The high working frequency of DSP enable it. The diagram of the program is shown in fig.7.

\section{C. motor position controlling program}

The motor position controlling program is the core of the whole program. In the program, each motor's position is compared with it's predefined position, then the program give corresponding signal to drive the motor to run forward or reverse. The controlling mode is closed-loop control. The feedback signal of position is analog voltage obtained from AD sampling program.

In order to finish each controlling process quickly and accurately, the closed-loop control policy is PID.

In PID mode, the parameter of proportation (Kp) affects stability and speedability of dynamic state process . A bigger Kp can make the speedability better, but cause the stability bad at same time. A bigger $\mathrm{Kp}$ can increase times of oscillation. It causes the motor to shake, then decrease the service life of motor. In my actual work, the $\mathrm{Kp}$ is determined by calculation, simulation and experiment.

A integral can eliminate the steady-state error of system, then crease the accuracy of steady-state. But it needs more complex calculation and causes the stability bad. According to precision index of steady-state, integral is not used in the system.

A differential affects dynamic performance only. The differential parameter (Td) is bigger, the overshoot of system is bigger and the adjustment time is shortened. When Td is smaller, the adjustment time is get longer, but the overshoot of system is bigger as before. A appropriate differential parameter (Td) should be determined to obtain a smaller overshoot and a shorter adjustment time. The $\mathrm{Td}$ is determined by calculation, simulation and experiment too. The diagram of the program is shown in fig.8.

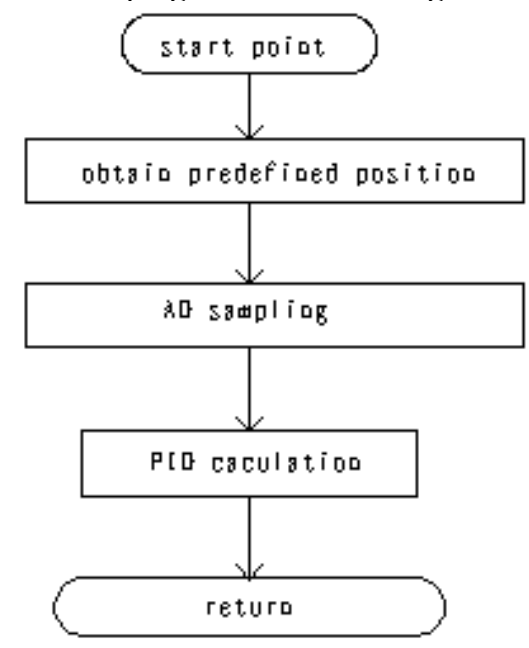

(fig.8 diagram of position controlling)

\section{RS485 communication program}

The RS485 communication program is in charge of communicating with upper computer. The communication protocol is MODBUS which is widely used to industrial control. It uses CRC checkout, and has advantages such as brief instructions, high efficiency and high reliability.

The datas of SCI are queried in fixed interval time which is 625us in the program. Put the datas obtained together continuely and check its completeness. When completeness and CRC are all right, a flag bit is set to indicate the datas valid.

\section{IV.THE CONCLUSIONS}

The paper introduces a control system for ink keys of press based on DSP controller TMS320F2812. The system has advantages such as high speed calculation, high accuracy, high integrity, simple hardware circuit and good electromagnetic compatibility. The operators judge that the actual presses fitted the system are more convenient and more accurate than normal presses. The results obtained from real operation indicates that the PID control mode and the optimizational digital filter can control the ink thickness efficiently.

\section{REFERENCES}

[1]Su Yugang,"The Design and Development of Remoted Ink Fountain Control System”,Beijing, 2008.3-46

[2]Zhang Weining,"The CPU and Peripherals of TMS320C28X series DSP(2Th)",beijing, Tsinghua University Press, 2005.349-376

[3] TI.TMS320F28X Analog-to-digital Converter (A/D) Reference Guide[M].Dallas: Texas Instruments Inc.July 2005.1-60

[4]Zhang Weining,"The CPU and Peripherals of TMS320C28X series DSP(1Th)”,beijing, Tsinghua University Press, 2004.121-196

[5] TI.TMS320C28x Optimizing C/C++ Compiler User's Guide[M]. Dallas: Texas Instruments Inc. October 2005.72-100, 125-162

[6] TI.TMS320C1x/2x/2xx/5x Assembly Language Tools User’s Guide[M]. Dallas: Texas Instruments Inc. 1995 